\title{
Active Immunotherapy in cancer - Current Status
}

\author{
Bakulesh Khamar* \\ R\&D Department, Cadila Pharmaceuticals Limited, India
}

*Corresponding author: Bakulesh Khamar, R\&D Department, Cadila Pharmaceuticals Limited, "Cadila Corporate Campus, Sarkhej - Dholka Road, Bhat, Ahmedabad, Gujarat, India-382210

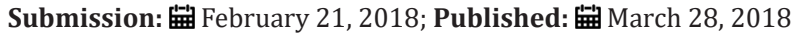

\begin{abstract}
Immunotherapy is a fourth pillar in cancer management after surgery, radiotherapy and chemotherapy. Active immunotherapy works by inducing antigen specific immune response following its administration while checkpoint inhibitor works by reducing tumor induced immunosuppression and thereby utilizing pre-existing immune response. Adoptive cell therapy (ACT) is a type of personalized therapy wherein cells are harvested from a patient, expanded and reintroduced. The T cells are genetically modified in chimeric antigen receptor T cell (CAR-T) therapy while tumor infiltrating cells are used in in ACT and dendritic cells are used in dendritic cell (DC) vaccine. Vaccine induces T cell response against the antigen of interest. T cells available following single administration are higher in ACT compared to vaccine approach. CAR-T cells are found useful in refractory large B-cell lymphoma and refractory large B-cell precursor acute lymphoblastic leukemia. ACT is found useful in melanoma. Vaccines are approved for melanoma as a monotherapy and for non-small cell lung cancer in combination with chemotherapy.
\end{abstract}

Keywords: CAR-T therapy; ACT; Dendritic cell vaccine; Cancer vaccine; CD8+T cells; Active immunotherapy

\section{Introduction}

Cancer development and progression is associated with inability of immune system to recognize cancer cells as foreign and destroy it. This is done mainly by cell mediated immune response. Cells mediated immune response play a critical role in development and progression of cancer. Effective utilization of cell mediated immune response holds promise to cure cancer. Early evidence indicating importance of immune cells came from histopathological evaluation of tumor infiltrating immune cells and their role in prognosis [1-7]. It suggests correlation between amount and type of immune cells with outcome is well documented [3,7-13]. Increase in Th1-type signature is associated with good clinical outcome in many different tumor types including colorectal cancer, melanoma, head \& neck, breast, bladder, urothelial, ovarian, renal, prostate, hepatocellular cancer and lung cancer $[10,14,15]$. CD8 cell preponderance is associated with better outcome [12,1618]. CD45RO + $\mathrm{T}$ cells infiltration is known to improve outcome in esophageal (also squamous) cancer, colorectal cancer, ovarian cancer, gastric cancer, hepatocellular cancer [19].

NK cell infiltration is also described as a positive prognostic factor [20]. Preponderance of immunosuppressive immune cells like FOXP3+ Treg, MDSCs are associated with poor prognosis including worse OS [17,21-25]. Relative proportion of either Tregs or MDCSs cells (immunosuppressive cells) to CD8+T effector cells is a strong prognostic indicator for responses to chemotherapy/ immunotherapy and patient outcomes [26].
Recognizing importance of immune mechanism in cancer, "Immunoscore," is designed for better prognostication. It is found to superior to the conventional TNM classification in colorectal cancer, Non- small cell lung cancer (NSCLC), hepatocellular cancer and gastric cancer [27-32]. In NSCLC immunoscore using CD45RO+ cells and CD8+T cells predict outcome in Squamous NSCLC but not in adenocarcinoma. In Squamous NSCLC higher score is associated with better outcome compared to lower score $(\mathrm{P}<0.001)$. The hazard ratio (HR)/ (benefit) increases with the stage of disease. It is $4.35(\mathrm{p}=0.012)$ for stage I, $8.24(\mathrm{p}<0.001)$ for stage II and 9.52 $(p=0.002)$ for stage III [19]. Immunoscore is associated with [33].

I. Rate of response to treatment

II. Survival in patient

III. Responding to treatment

IV. Not responding to treatment

Based on presence or absence of Tumor Infiltrating lymphocytes (TIL) and checkpoint protein tumor classification is proposed. Tumors without TIL and checkpoint protein may benefit from active immunotherapy while tumors with checkpoint protein may benefit from checkpoint inhibitor. Both may be synergistic when TIL is absent and checkpoint protein is present or has a potential following active immunotherapy [6]. 
The importance of immunotherapy became evident and came to limelight with approval of checkpoint inhibitors. Immunotherapy is now considered fourth pillar of cancer management following surgery, radiotherapy, chemotherapy. Approved checkpoint inhibitors work by on immune suppressive arm by removing barriers to $\mathrm{T}$ cell activation to enhance preexisting immune response. Active immune therapy in general works by activating host immune response to a particular antigen/s. Such an immune response is also known as adaptive immunity and is provided by lymphocytes. Each person possesses over 1011 lymphocytes [34] with T-cells making around $80 \%$ of it. T cells possess receptors to recognize antigen and generate adaptive immune response against it. To generate response $\mathrm{T}$ cells needs co-stimulation (second signal) besides antigen recognition (first signal).

In any case, the correlation between macrophage density and patient survival is less significant than that of $\mathrm{T}$ cells, particularly CD8+T cells [16].

Approval of Axicabtagene ciloleucel for large B cell lymphoma in adults and tisagenlecleucel for Refractory large B-cell precursor acute lymphoblastic leukemia in 2017 by US FDA has rekindled interest in active immunotherapy. Both are personalized CAR Tcell therapy. Both provide objective response rate (ORR) of more than 80\% (Axicabtagene ciloleucel - 82\% [35] and tisagenlecleucel - 81\% [36]) which seems to be durable like checkpoint inhibitor at this early stage.

Efficacy of Active Immunotherapy Depends on Antigen, Effectors Function and Tumor Micro Environment

\section{Antigens}

Antigens are substances (mainly proteins, polysaccharide, and peptides) found in tumor cells which are capable of generating activation (first) signal required for cell mediated immune response. Absence of antigen expression by tumor is associated with lack of response. Whole cells, cell lysates are also used to provide antigens. $T$ cell infiltration is required for the efficacy of immunotherapy proportional to antigen expression [37]. Antigen expression is decreased by deacetylation or hypermethylation and can be increased by use of appropriate therapeutic agent like azacitidine [37].

Loss of antigen expression is associated with adaptive resistance to active immunotherapy as seen with CAR-T cell therapy [36]. Antigens expressed by tumors can be classified into following major groups $[5,34]$.

Over expressed normal proteins: They are also named Tumor associated antigen. They are usually expressed at low levels in normal tissues but over expressed in cancer tissues (e.g., HER2, carcinoembryonic antigen (CEA) or non-mutated p53); nonmutated differentiation antigens (e.g., MART-1, over expressed in melanoma and found in normal melanocytes but not in other cells)

Cancer-testis antigens (CTA): CTA are expressed by non- mutated genes during fetal development, then silent in normal adult tissues and reactivated in cancer cells across multiple malignancies (e.g., MAGE and NY-ESO).

Mutated antigens or neoantigens: They are also called tumor specific antigens and are found only in tumors [38]. They are caused by non-synonymous mutations or encoded by viral genes in tumors of viral origin and are unique to a single tumor or shared by a group of tumors (e.g., BRAF with the V600E mutation in melanoma and other solid tumors, or EGFR vIII in glioblastoma).

\section{Effector function}

Effect or function measures strength of cell mediated immune response to kill tumor cells. It can be performed ex-vivo or in-vivo. It depends on type of immune cells, number of immune cells, affinity of immune cells and duration for which immune cells are available.

Type of immune cells: Activated antigen specific CD8+ cells are mainly responsible for killing of tumor cells [39]. CD4+ cells provide help to CD8 cell. They have minimal potential for killing tumor cells. $\mathrm{T}$ cells in earlier stage of differentiation have better efficacy and resistance in tumor compared to terminally differentiated [37].

No. of immune cells: concentration of activated antigen specific CD8+ T cells determines the efficiency with which these cells kill antigen-expressing target cells [39]. Increase in no. of CD8+ cells post therapy is associated with efficacy of vaccine [39] as well as its combination with checkpoint inhibitors [38]. A critical concentration of activated antigen specific CD8+ T cells in relation to tumor burden is required for efficacy [39]. Responders to tisagenlecleucel (CAR T-cells) had higher Cmax 36,100 copies /mcg of CAR T-cells compared to nonresponders 20,900 copies/mcg [36]. Fold change in activated antigen specific CD8+ T cells in relation to tumor burden following therapy is associated better ORR, PFS, and OS even with checkpoint inhibitors [23].

Affinity of immune cells towards antigen bearing tumor cells: Besides type and number of cells, affinity of activated antigen specific immune cells determine killing kinetic of immune cells in tumor bed [38]. Low affinity is associated with poor outcome [40]. It also depends on amount and type of antigen expressed by tumor cells. Tumors with low immunogenicity are counterproductive [40].

Duration for which immune cells are available/persistence: Duration for which activated antigen specific immune cells are available is also important for response to active immunotherapy [39], [40]. Longer duration increases chance of response. With tisagenlecleucel (CAR T-cells), T1/2 in responders was 23.0days compared to 3.6days for non-responders [40]. T last median was 168days for responder's vs 49 days for non-responders [40].

Time to achieve peak no. of cells: Shorter time is associated with better prognosis compared to longer time [36]. With tisagenlecleucel (CAR T-cells) Tmax of 10days was associated with response to therapy while Tmax of 20days was seen in nonresponders [36]. 


\section{Tumor microenvironment}

Hostile tumor microenvironment (TME) to anti-tumor immune response is associated with tumor growth and its spread. TME comprise of cancer cells, tumor educated stromal cells, infiltrating immune cells and tumor vasculature. Cancer cells play a major role in initiation of immunosuppressive changes in initial stages of cancer development which is maintained and potentiated by stromal cells and infiltrating immune cells. Immune changes brought about by cancer cell include (a) impaired T-cell immunologic synapse function through $\mathrm{F}$ actin [41] (b) apoptosis of T cell and (c) promotion of Treg by tumor exosome [42]. Cancer cells change profile of stromal fibroblasts to cancer associated fibroblasts (CAF). CAF are major component of stroma and account for more than $90 \%$ of cells in tumors like pancreatic cancer [43]. CAF from advanced tumor upregulates TGF-beta and induces immunosuppressive environment including recruitment of immune cells and their conversion to immunosuppressive cells [44]. CAF are also involved in metastasis [45]. They secrete angiogenesis factors like VEGF, FGF, SDF-1 and PDGF [46]. CAF resist killing of tumor cells by various therapeutic agents by [46] including (a) expression of MMP1, MMP2, MMP9, BCL-XL. (b) providing support to stem cells (c) inducing low $\mathrm{pH}$ of environment, (d) increased hyaluronan production, (e) increased interstitial fluid pressure etc. of CAF in stroma is inversely proportional to survival in colorectal cancer [46].

Cancer cells are known to divide rapidly, have very high cell density and high metabolic rate with relative hypoxia leading acidic microenvironment as tumor increases in size. Changes in immune profile $[42,47]$ due to low $\mathrm{pH}$ include (a) loss of cytolytic activity by tumor specific cell: $\mathrm{T}$ and NK cells tend to lose their function and undergo a state of mostly reversible energy followed by apoptosis, (b) accumulation of immunosuppressive cells like myeloid cells and regulatory T cells, (c) secretion of immunosuppressive cytokines.

Tumor is highly vascular but vasculature is known to dysfunctional and limits exchange beyond tumor including infiltration of immune cells and cytokines. Thus cancer cells through their direct and effect on immune cells and through changes in stroma is associated with immune suppression making it difficult for active immunotherapy to be effective in cancer management.

In general a solid tumor like melanoma contains 3-5x108cells/ gm [48] and for its eradication $>107$ melanoma (tumor) antigen specific CD8 cells/g of melanoma for $>8$ days is required [39].

Generating such a large number of antigen activated CD8 cells within body is a herculean task and is one of the reason for absence of tumor response with vaccine approach in established tumors. To overcome limitation, immune cells are expanded in vitro prior to reintroduction in a personalized approach. The amount of CAR T- cells infused is $>108$ for adults and for tumor infiltrating cells is for vaccine approach to be successful, it needs support from other therapy in management of advanced tumors [34]

\section{Active immunotherapy comprises mainly two types of therapy}

1. Large no. of activated immune cells are introduced as a therapy. This is currently a personalized approach and comprise of autofocus cell transfer after expansion

\section{CAR T-cell therapy}

\section{Adoptive cell transfer (ACT) of tumor-infiltrating lymphocytes (TILs)}

\section{Dendritic cell vaccine}

2. Vaccines

Approved Products

A. T-VEC

B. CADI-05

3. Their Vaccine candidates

A. Allogenic cell based vaccine

B. Protein/peptide based vaccines

C. Viral vector based vaccines

\section{CAR T-cell therapy}

The chimeric antigen receptor $\mathrm{T}$ cell (CAR-T) therapy involves leukapheresis for the removal of peripheral-blood $\mathrm{T}$ cells from a patient, followed by in vitro activation, genetic modification to express a Chimeric Antigen Receptor (CAR) on their cell membrane, and expansion of the $\mathrm{T}$ cells under Good Manufacturing Practice conditions, and finally the infusion of the cells back into the patient so they will attack cancer cells [49]. CAR-T cells are expected specifically to localize within tumor and eliminate tumor cells by interacting with the antigens expressed on tumor cell surface. First generation CAR-T had antigen to induce activation signal and provided transient $\mathrm{T}$ cell activation. Subsequent CAR-T cells also induces co-stimulatory signal [50].

\section{Table 1}

\begin{tabular}{|c|c|c|c|}
\hline & ORR & CR \\
\hline $\begin{array}{c}\text { Refractory large B-cell } \\
\text { lymphoma }\end{array}$ & $\begin{array}{c}\text { Axicabtagene ciloleucel } \\
{[51]}\end{array}$ & $82 \%$ & $58 \%$ \\
\hline $\begin{array}{c}\text { Refractory large B-cell } \\
\text { lymphoma }\end{array}$ & CTL 019 [52] & $63 \%$ & $57 \%$ \\
\hline $\begin{array}{c}\text { Refractory large b } \\
\text { cell precursor acute } \\
\text { lymphoblastic leukemia }\end{array}$ & Tisagenlecleucel [40] & $81 \%$ & $60 \%$ \\
\hline
\end{tabular}

Axicabtagene ciloleucel and tisagenlecleucel are approved CAR T-cells [49]. Both contain CD19 directed genetically modified autologous $\mathrm{T}$ cells to express second-generation anti-CD19 CARs. CD19 is an antigen found in B-cell malignancies, which include several forms of lymphoma and leukemia. Major difference between two is costimulatory domain. In axicabtagene ciloleucel 
costimulatory domain is C28 while it is 4-1BB in tisagenlecleucel. CTL019 is another advanced CAR T-cell therapy evaluated for B cell lymphoma. CAR T-cell therapy achieves complete response in more than $55 \%$ of treated patients (Table 1).

Unlike checkpoint inhibitors, CAR T-cell therapy (activated antigen specific cells) is administered once and it quickly induces response. Axicabtagene ciloleucel induces response with a median time to response - 1 month (range 0.8 - 6 months) [51]. Partial responses are not durable (2.1 month) but complete responses seems to be durable (Label - FDA). OS with tisagenlecleucel is $90 \%$ at 6 months $76 \%$ at 12 months again indicating durability of response [40]. Recurrence is associated with loss of antigen (CD19) in 15 of 22 indicating loss of antigen as a major cause for acquired immune resistance. Both approved CAR T- cells and CAR T-cells in general are associated with severe side-effects, such as neurotoxicity and cytokine release syndrome leading to death. A neurologic toxic effect most commonly includes encephalopathy, confusion, and tremor. The cytokine-release syndrome most commonly includes fever, hypoxia, and hypotension. Cytokine storm is attributed to large no. immune cells infused. The majority of these side effects are reversible, with no clinical sequelae.

Attempts are made to develop improved CAR T cells with reduced side effect or efficacy.

A. Reducing side effects: Switchable CAR T cells and use of gamma delta $\mathrm{T}$ cells as autologous cells to produce CAR T-cell appear most promising CAR T cells with reduced side effect.

I. Switchable CAR T cells: Switchable CAR T cells are being evaluated to decrease on target, off tumor side effect profile. The Switch molecules are comprised of tumor targeting antibody or small molecule and second moiety which selectively binds to CAR and not endogenous receptor. Switchable CAR T cells are activated by administration of tumor targeting antibody or a small molecule $[53,54]$. Rapamycin and tacrolimus are two small molecules being evaluated [55]. CAR T cells get activated in presence of rapamycin. They are not activated when rapamycin is not available.

II. CAR T cells with gamma delta T cells: Use of gamma delta $\mathrm{T}$ cells , instead of alpha beta T cells, to generate CAR T cells is being evaluated [56,57]. Gamma delta $\mathrm{T}$ cells possess unique features such as direct antigen recognition, lack of alloreactivity, and ability to present antigens [57]. They are expected to have enhance cytotoxicity while retaining their ability to migrate to tumor and act as antigen-presenting cells to prolong the intratumoral immune response [56]. They are expected to have reduced side effect profile as they selectively target cancer cells without attacking healthy cells. They might find use in management of solid tumors as they can present antigens.

\section{B. Improving efficacy}

I. Overcoming immunosuppression: Persisting CAR-T cells following infusion of CAR-T cells, have decreased activity compared to pre-infusion CAR-T [58, 59]. Decreased activity is associated with expression of pd-1 and co-inhibitory receptor
CD160. Expressions of immunosuppressive markers are also associated with primary and secondary failure of CAR-T therapy. They are also considered main reason for poor response in chronic lymphocytic leukemia and solid tumors. Combinations with check point inhibitors are being evaluated. Efforts are made to use CRISP technology to incorporate checkpoint inhibitor into CAR-T cells [60] or generate CAR-T cells which do not express PD-1 under any circumstances [61]. CAR-T cells targeting to T-cell receptor alpha constant locus. TRAC -CAR-T cells Current production technology for CAR T-cells production is associated with suboptimal yield and weak activity. They decrease in no. over time and have increased exhaustion markers in persistent cells [58] resulting in decreased efficacy. TRAC-CAR T-cells have uniform CAR expression in peripheral blood $\mathrm{T}$ cells and better efficacy resulting in better tumor control (greater response and prolonged median survival) in animal studies [62]. This is associated with persistence of activated TRAC-CAR T cells over time and absence of de-novo expression of exhaustion markers (2\% vs $50 \%$ on day 17 ) [62].

\section{Scalable of the shelf product}

Current therapy needs autologous T cells to be harvested, modified and expanded, making this therapy time and resource consuming. Efforts are made to use allogenic T cells so that therapy can be made readily available. The challenge is to avoid graft vs. host disease. CRISPR technology is evaluated for knocking out the TCR and HLA genes to bypass GvHD [59].

D. CAR-T cells for solid tumors: Currently CAR T-cell therapy is limited to hematological malignancyx

i. NKR-2: NKR-2 are autologous T cells genetically modified to express a chimeric antigen receptor (CAR) comprising a fusion of the natural killer group 2D (NKG2D) receptor with the CD33 signaling [63]. It binds eight different ligands expressed on the cell surface of above $80 \%$ of solid and hematological malignancies which are normally absent on non-neoplastic cells. In preclinical studies, NKR-2 demonstrated long-term antitumor activity towards a breadth of tumor indications, with maximum efficacy observed after multiple NKR-2 administrations. Importantly, NKR2 targeted tumors cells and tumor neovasculature and the local tumor immunosuppressive microenvironment. It does not need preconditioning.

ii. Anti TAA (tumor associated antigen) CAR T cells: Efforts are made to target tumor associated antigens like EGFR, human epidermal growth factor receptor 2 (HER2), mesothelin (MSLN) MUC 16etch for use in solid tumors [63]. In early clinical trials they appear safe and able to generate immune response. The response rate is not good [63]. This can be attributed to lack of TAA, inefficient trafficking of CAR-T cells, hostile micro environment.

Adoptive cell transfer therapy (ACT) of tumor-infiltrating lymphocytes (TILs)

Some TILs exhibits specific tumor lysis ability without killing normal cells both in vitro and in vivo. TILs are predominantly T cells with heterogeneous phenotypes that contained effector cells, and 
memory cells, with most of them possessing specific tumor antigen recognizing ability. Adoptive cell transfer (ACT) of tumor-infiltrating lymphocytes (TILs) involves isolation of TILs with antitumor activity, their expansion and re-infusion. [64]. Lymphodepletion before ACT is an important component of the treatment because it eliminates $\mathrm{T}$ regulatory cells and eliminates lymphocytes, which compete with the transferred cells for homeostatic cytokines such as interleukin 7 (IL7) and IL15 [64].

Current culture technique for $\mathrm{T}$ cell expansion allows, more than 1011 cells to be transfused $[64,65]$. Clonal repopulation of $\mathrm{T}$ cells directed against over expressed self-derived differentiation antigens, in combination with chemotherapy and high doses of IL2 , led to tumor regression in patients with metastatic melanoma [66]. Following infusion, TIL initially accumulates in the lungs for up to $24 \mathrm{~h}$ and then gets deposited in tumor sites [67].

Clinical responses are associated with (a) amount of tumorresident CD8+ $\mathrm{T}$ lymphocytes targeting tumor-specific antigens $[68,69]$ and depends on [70] (b) transfusion of younger phenotype cells [65], (c) cells with shorter doubling times, (d) higher cytotoxic capacity [71] or (e) higher GM-CSF secretion [65,72]. Nevertheless, analyses of neoantigen specific T-cell responses in melanoma patients treated by ACT demonstrated that the T-cell-recognized neoantigens can be selectively lost over time emphasizing the importance of targeting broad TCR recognized neoantigens to avoid tumor resistance [73].

ACT of TIL is mainly used for treatment of melanoma. It is possible to achieve ORR of 50\% [64] and CR rates of $24 \%$ in both groups and median OS of 38.2 months [74].

It is also used in treatment of other tumors like colorectal cancer [65], hepatocellular cancer [75] with good outcome. Progression or recurrence is associated with loss of antigen expression by tumor [65].

The use of donor lymphocytes for ACT is an effective treatment for immunosuppressed patients who develop post-transplant lymphomas [64].

Application of this therapy to other solid tumors is always not possible as some tumors, "immune-desert tumors" or "cold tumors," may have no or occasional TIL or TIL may display an exhausted state.

\section{Dendritic cell (DC) vaccines}

This is a personalized vaccine approach using extremely efficient antigen presenting properties of dendritic cells for induction of T cell immunity. In this approach, DCs are isolated from the patients' peripheral blood mononuclear cells (PBMC), loaded with tumor antigens ex vivo, activated, and then re-infused back into the patient [76-78]. This approach has been evaluated in various academic centers for management of advanced cancers with some modest improvement in outcome. Sipuleucel-T is the first dendritic vaccine approved by US FDA in 2010 for treatment of metastatic prostate cancer as it improved survival by four months without response (tumor shrinkage) in any patients. The vaccine is prepared by culturing dendritic cells isolated from patients with a fusion protein PA2024, consisting of PAP (Protein found in prostate cancer but nit in normal prostate tumors) linked to a granulocytemacrophage colony-stimulating factor (PAP-GM-CSF). These activated APCs are eventually infused back into the patient.

Vaccine

\section{A. Approved Products}

I. Talimogene laherparepvec (T-vec) (Oncolytic Virus Vaccine): Oncolytic virus vaccine uses Oncolytic viruses which are native or attenuated viruses that selectively replicate in cancer cells lyse them and induce host antitumor immunity [79]. It harbors low levels of protein kinase R (PKR) and dysfunctional type-I IFN signaling elements [79]. It generates tumor-specific Immunity immune response probably by direct tumor cell lysis, release of soluble tumor antigens, and danger-associated molecular factors.

Talimogene laherparepvec (T-vec) contains genetically modified herpes simplex virus, type I and is approved for the local treatment of unresectable cutaneous, subcutaneous, and nodal lesions in patients with melanoma recurrent after initial surgery. Its administration is associated with increase in activated CD8+ T cells (CD3+, CD4-, HLA-DR+) 25-28 in peripheral from baseline by 1.51fold, (4 weeks), 1.65-fold, (6 weeks), respectively [80]. Increases in activated CD8+ T cells seemed to correspond with patient response [80].

Efficacy and safety: Durable response rate (DRR), defined as the percent of patients with complete response (CR) or partial response (PR) maintained continuously for a minimum of 6 months, was $16.3 \%$ [81]. The median time to response was 4.1 (range: 1.2 to 16.7) months. T-VEC efficacy decreases with advanced disease stage IIIB, IIIC is $33 \%$ and for IVM1a is $16 \%$. It is $24 \%$ for treatment-naive patients. T-VEC resulted in complete resolution of lesions in $47 \%$ of injected lesions, $22 \%$ of uninjected non-visceral lesions and 9 $\%$ of visceral lesions [82]. Median OS was 23.3 months with T-VEC versus 18.9 months with GM-CSF (hazard ratio, 0.79; 95\% CI, 0.62 to $1.00 ; \mathrm{P}=.051$ ) with better OS in patients with stage IIIB, IIIC, or IVM1a disease (HR, $0.57 ; 95 \% \mathrm{CI}, 0.40$ to 0.80 ) and previously untreated patients (HR, 0.50; $95 \% \mathrm{CI}, 0.35$ to 0.73 ) [81]. The most common adverse events (AEs) with T-VEC were fatigue, chills, and pyrexia. The only grade 3 or $4 \mathrm{AE}$ occurring in $\geq 2 \%$ of T-VECtreated patients was cellulitis (2.1\%) [81].

\section{Combination with checkpoint inhibitors:}

Iplimumab: Combining it with iplimumab improved response rate (irRC) to $50 \%$ (95\% CI, 26.0 to 74.0 ) with DRR in $44 \%$ and CR in Four patients (22\%) [80]. Responses were seen $74 \%$ of injected and $52 \%$ of uninjected [80]. Complete response was identical in both groups (31\% vs.39\%) [80].

Pembrolizumab: Combination therapy with pembrolizumab [83] improved objective response rate to $62 \%$, with a complete response rate of $33 \%$. Patients who responded to combination 
therapy had increased CD8+T cells, elevated PD-L1 protein expression, as well as IFN- $\gamma$ gene expression on several cell subsets in tumors after talimogene laherparepvec treatment [83]. Response do not appear to be associated with baseline CD8+ T cell infiltration or baseline IFN- $\gamma$ signature [83].

Response to therapy is better with combination therapy. It is best with anti PD-1 therapy. These findings suggest that oncolytic virotherapy may improve the efficacy of anti-PD-1 therapy by changing the tumor microenvironment [83]. Some of the viruses being evaluated in various cancers as virus vaccine include [79]:

a. Adenovirus: Bladder cancer, ovarian cancer, prostate cancer, head and neck cancer, sarcomas, NSCLC, glioblastoma

b. Coxsackie virus : Melanoma, breast cancer, prostate cancer

c. HSV-1: Melanoma, breast cancer, head and neck cancer, pancreatic cancer

d. Measles virus : Ovarian cancer, glioblastoma, multiple myeloma

e. Newcastle disease virus :Glioblastoma Type I IFN and Bcl-2

f. Parvovirus: Glioblastoma

g. Poliovirus: Glioblastoma

h. Poxvirus: Head and neck cancer, hepatocellular carcinoma, melanoma, colorectal cancer

i. Reovirus: NSCLC, ovarian cancer, melanoma, head and neck cancer

j. Seneca valley virus: Neuroblastoma, lung cancer

k. Vesicular stomatitis: virus Hepatocellular carcinoma

\section{CADI-05}

CADI-05 induces cell mediated immune response against Desmocollin-3 (DSC3) expressing tumors [84,85]. DSC3 expression is used to differentiate Squamous NSCLC from adeno variety of NSCLC [86]. It is also expressed by ovarian cancer, melanoma, colorectal cancer, bladder cancer and [87]. In DSC3 negative epithelial tumors, it can be expressed by appropriate therapy like radiotherapy, chemotherapy, targeted therapy [87]. CADI-05 induces antitumor response through IFN-gamma secreting CD8+Tcells [88] by increasing tumor infiltrating activated immune cells and reducing intratumoral immunosuppressive cells [89].

In randomized control clinical trial in advanced NSCLC, its combination with chemotherapy, it improved response rate by $10 \%$ ( $47 \%$ vs $37 \%$ ) with the improved os by $17.48 \%$ at the end of 1 year [84]. The benefit (Hazard ratio) more marked in patients with better performance ( 0.51 for ECOG0 vs 0.87 for ECOG1), less advanced disease ( 0.580 for stage IIIB vs 0.705 for stage IV), histology ( 0.40 for Squamous NSCLC vs 0.69 for adeno NSCLC) and seems to be related to amount of chemotherapy received. No benefit was seen in nonresponders.

CADI-05 achieves and maintains remission in melanoma as well as in bladder cancer as a systemic monotherapy [90,91]. It achieves complete remission in muscle invasive bladder cancer when combined with radiotherapy which does not recur at least for two years [92].

It is approved in India for treatment of NSCLC and has orphan drug designation from US FDA for DSC3 expressing NSCLC.

\section{B. Other Vaccine Candidates}

I. Allogenic cell based vaccine

a. Gvax: An irradiated, syngeneic, GM-CSF-expressing tumor-cell vaccine (Gvax) has been shown to evoke dense intratumoral infiltrates of APCs displaying superior antigenpresenting activity. GVAX Pancreas induces large immunoglobulin $G$ and immunoglobulin $M$ responses to many antigens, including tumor-associated carbohydrates, blood group antigens, $\alpha$-Gal, and bovine fetuin [93]. Gvax melanoma is associated with significant increases in eosinophils and PD-1+ lymphocytes from cycle 1 to cycle $4(p<0.05)$ at vaccine site [b] Serum GM-CSF concentrations [a] increased numbers of activated circulating monocytes $(p<0.0001)$ [c] decreased percentages of myeloid-derived suppressor cells among monocytes (CD14+ , CD11b+ , HLA-DR low or negative; $p=0.002$ ) [94]. It failed when used as a standalone intervention against established tumors [95]. Phase III trials due to a lack of clinical efficacy. Among a myriad of potential reasons for this lack of success, one may be the presence of potent immune evasion mechanisms in established lesions. The failure could be attributed to inadequate immunogenicity of the approach and alterations in preparation of the vaccine product required by commercial scale-up [96]. Combination of a GVAX with an immune checkpoint blockade demonstrated no improvement over the blockade alone [97].

b. Belagenpumatucel-L: Belagenpumatucel- $L$ is a therapeutic vaccine comprised of 4 transforming growth factor (TGF)-b2-antisense gene-modified, irradiated, allogeneic NSCLC cell, for maintenance therapy in NSCLC. In a phase III trial, 532 Patients with stable disease or response following up to 6 cycles of a platinum-based frontline chemotherapy regimen, with or without radiation therapy were randomized to receive maintenance belagenpumatucel-L (2.5 107 cells per dose) or placebo in a 1:1 ratio [98]. There was no difference in PFS (4.3 months versus 4.0 for belagenpumatucel-L vs placebo, respectively; HR 0.99, $\mathrm{p}=$ 0.947 ) or OS between the arms (median survival 20.3 versus 17.8 months with belagenpumatucel-L versus placebo, respectively; hazard ratio (HR) $0.94, p=0.594$ ). Time to randomization after end of chemotherapy had a significant impact on survival ( $\mathrm{p}=$ 0.002). Benefit of treatment (HR 0.77, 95\% CI 0.56-1.05; $\mathrm{p}=$ 0.092 ) was seen in those who were randomized within 12 weeks of the completion of frontline chemotherapy (median OS 20.7 
months (95\% CI 14.6-26.9) vs 13.4 months (95\% CI 9.9-16.7). Prior chemora was a positive prognostic factor (median survival 28.4 months with belagenpumatucel-L versus 16.0 months with placebo; HR 0.61, $\mathrm{p}=0.032$ ).

c. Canvaxin: Canvaxin is an allogeneic whole-cell vaccine developed from three melanoma cell lines. It was evaluated in stage-III melanoma in a phase III trial [88]. It was discontinued based on the recommendation of the independent Data and Safety Monitoring Board (DSMB) with oversight responsibility for the clinical trial after review of the third interim analysis. Findings suggested it was unlikely that the trial would provide significant evidence of a survival benefit for Canvaxin-treated patients versus those receiving placebo. Trial evaluated three biomarker in peripheral blood MART-1, MAGE-A3 and PAX3 by measuring mRNA. Multivariate analysis suggest that absence of all three at base line as well as post treatment has better survival with HR for OS 1.53 (95\% CI 1.05-2.24; $\mathrm{p}=0.028)$, HR for PFS 1.64 (95\% CI 1.19-2.24 $p=0.002$ ) for pretreatment Number of biomarkers $(+)>0$ vs. 0 . For post-treatment Number of biomarkers $(+)>0$ vs. 0 HR for OS was 2.57 (95\% CI 1.23-5.36; $\mathrm{p}=0.012$ ) and for PFS was 1.91 (95\% CI 1.11-3.; $\mathrm{p}=0.020$ )

\section{Protein/peptide based vaccines}

a. IMA901: IMA901, a vaccine consisting of ten tumorassociated peptides induces T-cell response. The IMPRINT study is an open-label, randomized, controlled, phase 3 trial [99]. 339 HLA-A*02-positive patients (aged $\geq 18$ years) with treatmentnaive, histologically confirmed metastatic or locally advanced (or both) clear-cell renal cell carcinoma were randomly assigned (3:2) to receive sunitinib plus IMA901 (4.13mg) and granulocyte macrophage colony-stimulating factor $(75 \mu \mathrm{g})$, with one dose of cyclophosphamide $(300 \mathrm{mg} / \mathrm{m} 2) 3$ days before the first vaccination $(n=204)$, or to receive sunitinib alone $(n=135)$. Sunitinib (50mg) was given orally once daily, with each cycle defined as 4 weeks on treatment followed by 2 weeks off treatment, until progression of disease. Median overall survival did not differ significantly between the groups $(33 \bullet 17$ months [95\% CI 27•81-41•36] in the sunitinib plus IMA901 group vs not reached [33•67-not reached] in the sunitinib monotherapy group; hazard ratio 1•34 [0•96-1•86]; $\mathrm{p}=0 \bullet 087$ ). IMA901 did not improve overall survival when added to sunitinib as first-line treatment in patients with metastatic renal cell carcinoma.

b. Tecemotide: The START trial randomized 1,513 patients with stage III NSCLC who had completed chemoradiotherapy without progressive disease to tecemotide (L-BLP25), a vaccine against MUC1 (a transmembrane mucin family protein), or placebo. There was no significant difference in survival between the two arms (median OS of 25.6 months with tecemotide versus 22.3 months with placebo), Benefit for the vaccine in patients who received concurrent rather than sequential chemotherapy (30.8 vs. 20.6 months, $\mathrm{p}=0.0175$ ) [100].These promising results were not confirmed in a subsequent study [101] (median 32.4 versus
32.2 months, hazard ratio $0.95,95 \%$ confidence interval $0.61-1.48$; $\mathrm{P}=0.83$ ).

c. MAGE-A3 peptide based vaccine: Vaccine to melanomaassociated antigen 3 (MAGE-A3) induces MAGE-A3 specific CD4+T cell response in some and MAGE-A3 specific CD8+T cell response in few patients with respected NSCLC in presence or absence of chemotherapy [102]. In the MAGRIT trial, patients with surgically resected stage IB-IIIA MAGE-A3-positive NSCLC were randomized to receive placebo or a vaccine to MAGE-A3. A total of 2,272 patients were randomized and treated. All patients in vaccine group achieved seropositivity for anti-MAGE-A3 antibodies indicating immune response to vaccine. The trial showed no significant improvement in disease-free survival (60.5 versus 57.9 months, $p=0.7379$ ) [103].

\section{Viral vector based vaccines}

a. Tricom: Tricom vaccine comprises of vaccinia and fowlpox viruses encoding prostate-specific antigen (PSA) along with three costimulatory molecules: B7.1, intercellular adhesion molecule 1 (ICAM-1), and lymphocyte function-associated antigen 3 (LFA-3). It demonstrated a benefit in overall survival without evidence of tumor shrinkage and change in progression-free survival (median survival of 25.1 months, vs 16.6 -months, $p=0.015$ ).in patients with castration-resistant prostate cancer [104].

b. TG4010: TG4010 is a recombinant modified vaccinia Ankara that codes for MUC1 and interleukin-2 [105]. MUC1 encoded by TG4010 shares epitopes with tumor-associated MUC1. It is being evaluated in the phase IIb/III trial The TIME trial in combination with first-line chemotherapy. It uses biomarker TrPal to measure baseline natural killer activity. Phase IIb part of the trial demonstrates survival benefit (PFS, OS) in non-squamous NSCLC having baseline TrPal value of less than or equal to the Q3 (123 patients of 222 enrolled). HR for PFS 0.59 (0.40-0.87) $p=0.0033$ and OS 0.59(0.39-0.91) $\mathrm{p}=0.0072$ [105].

\section{Conclusion}

Active immunotherapy holds promise for curing cancer if appropriately used along with other therapies to overcome tumor induced immune suppression and to alter hostile tumor micro environment. A recent advance in active immunotherapy has resulted in approval of T-Vec for melanoma as a monotherapy and CADI-05 for non-small cell lung cancer in combination with chemotherapy.

\section{References}

1. Geukes Foppen MH, Donia M, Svane IM, Haanen JB (2015) Tumorinfiltrating lymphocytes for the treatment of metastatic cancer. Mol Oncol 9(10): 1918-1935.

2. Gentles AJ, Newman AM, Liu CL, Bratman SV, Feng W, et al. (2015) The prognostic landscape of genes and infiltrating immune cells across human cancers. Nat Med 21(8): 938-945.

3. Erdag G, Schaefer JT, Smolkin ME, Deacon DH, Shea SM, et al. (2012) Immunotype and immunohistologic characteristics of tumor-infiltrating immune cells are associated with clinical outcome in metastatic melanoma, Cancer Res 72(5): 1070-1080. 
4. Djenidi F, Adam J, Goubar A, Durgeau A, Meurice G, et al. (2015) CD8+CD103+ tumor-infiltrating lymphocytes are tumor-specific tissueresident memory $\mathrm{T}$ cells and a prognostic factor for survival in lung cancer patients. J Immunol 194(7): 3475-3486.

5. Sadozai H, Gruber T, Hunger RE, Schenk M (2017) Recent Successes and Future Directions in Immunotherapy of Cutaneous Melanoma. Front Immunol 8: 1617.

6. Teng MW, Ngiow SF, Ribas A, Smyth MJ (2015) Classifying Cancers Based on T-cell Infiltration and PD-L1. Cancer Res 75(11): 2139-2145.

7. Balermpas P, Michel Y, Wagenblast J, Seitz O, Weiss C, et al. (2014) Tumour-infiltrating lymphocytes predict response to definitive chemoradiotherapy in head and neck cancer. Br J Cancer 110(2): 501 509.

8. Uppaluri R, Dunn GP, Lewis JS (2008) Focus on TILs: prognostic significance of tumor infiltrating lymphocytes in head and neck cancers. Cancer Immun 8: 16 .

9. Fang J, Li X, Ma D, Liu X, Chen Y et al. (2017) Prognostic significance of tumor infiltrating immune cells in oral squamous cell carcinoma, BMC Cancer 17(1): 375.

10. Fridman WH, Pagès F, Sautès-Fridman C, Galon J (2012) The immune contexture in human tumours: impact on clinical outcome. Nat Rev Cancer 12(4): 298-306

11. Remark R, Becker C, Gomez JE, Damotte D, Dieu-Nosjean MC, et al. (2015) The non-small cell lung cancer immune contexture. A major determinant of tumor characteristics and patient outcome, Am J Respir Crit Care Med 191(4): 377-390.

12. Bremnes RM, Busund LT, Kilvær TL, Andersen S, Richardsen E, et al (2016) The Role of Tumor-Infiltrating Lymphocytes in Development, Progression, and Prognosis of Non-Small Cell Lung Cancer, J Thorac Oncol 11(6): 789-800.

13. Ibrahim EM, Al-Foheidi ME, Al-Mansour MM, Kazkaz GA (2014) The prognostic value of tumor-infiltrating lymphocytes in triple-negative breast cancer: a meta-analysis, Breast Cancer Res Treat 148(3): 467 476.

14. Angell H, Galon J (2013) From the immune contexture to the Immunoscore: the role of prognostic and predictive immune markers in cancer. Curr Opin Immunol 25(2): 261-267.

15. Sun C, Xu J, Song J, Liu C, Wang J, et al. The predictive value of centre tumour CD8+ $\mathrm{T}$ cells in patients with hepatocellular carcinoma: comparison with Immunoscore. Oncotarget 6(34): 35602-35615.

16. Bindea G, Mlecnik B, Tosolini M, Kirilovsky A, Waldner M, et al. (2013) Spatiotemporal dynamics of intratumoral immune cells reveal the immune landscape in human cancer. Immunity 39(4): 782-795.

17. Remark R, Becker C, Gomez JE, Damotte D, Dieu-Nosjean MC, et al (2015) The non-small cell lung cancer immune contexture. A major determinant of tumor characteristics and patient outcome, Am J Respir Crit Care Med 191(4): 377-390.

18. Schalper KA, Brown J, Carvajal-Hausdorf D, McLaughlin J, Velcheti V, et al. (2015) Objective measurement and clinical significance of TILs in non-small cell lung cancer. J Natl Cancer Inst 107(3).

19. Paulsen EE, Kilvaer T, Khanehkenari MR, Maurseth RJ, Al-Saad S, et al. (2015) CD45RO(+) Memory T Lymphocytes--a Candidate Marker for TNM-Immunoscore in Squamous Non-Small Cell Lung Cancer. Neoplasia 17(11): 839-848

20. Larsen SK, Gao Y, Basse PH (2014) NK cells in the tumor microenvironment Crit Rev Oncog 19(1-2): 91-105.

21. Gabrilovich DI, Ostrand-Rosenberg S, Bronte V (2012) Coordinated regulation of myeloid cells by tumours. Nat Rev Immunol 12(4): 253268.
22. Lewis CE, Pollard JW (2006) Distinct role of macrophages in different tumor microenvironments. Cancer Res 66(2): 605-612.

23. Huang AC, Postow MA, Orlowski RJ, Mick R, Bengsch B, et al. (2017) T-cell invigoration to tumour burden ratio associated with anti-PD-1 response. Nature 545(7652): 60-65.

24. Shang B, Liu Y, Jiang SJ, Liu Y (2015) Prognostic value of tumorinfiltrating FoxP3+ regulatory T cells in cancers: a systematic review and meta-analysis. Sci Rep 5: 15179.

25. Takenaka M, Seki N, Toh U, Hattori S, Kawahara A, et al. (2013) FOXP3 expression in tumor cells and tumor-infiltrating lymphocytes is associated with breast cancer prognosis, Mol Clin Oncol 1(4): 625-632.

26. Beavis PA, Slaney CY, Kershaw MH, Gyorki D, Neeson PJ (2016) Reprogramming the tumor microenvironment to enhance adoptive cellular therapy. Semin Immunol 28(1): 64-72.

27. Galon J, Pagès F, Marincola FM, Angell HK, Thurin M, et al. (2012) Cancer classification using the Immunoscore: a worldwide task force, J Transl Med 10: 205.

28. Mina M, Boldrini R, Citti A, Romania P, D’Alicandro V, et al. (2015) Tumorinfiltrating T lymphocytes improve clinical outcome of therapy-resistant neuroblastoma, Oncoimmunology 4(9): e1019981.

29. Wang M (2017) ImmunoScore predicts gastric cancer postsurgical outcome. Lancet Oncol 18(2): e68.

30. Ishii H, Azuma K, Kawahara A, Matsuo N, Tokito T, et al. (2017) Programmed cell death-ligand 1 expression and immunoscore in stage II and III non-small cell lung cancer patients receiving adjuvant chemotherapy. Oncotarget 8(37): 61618-61625.

31. Yao Q Bao X, Xue R, Liu H, Li J, et al. (2017) Prognostic value of immunoscore to identify mortality outcomes in adults with HBV-related primary hepatocellular carcinoma. Medicine (Baltimore) 96(17): e6735.

32. Mlecnik B, Bindea G, Angell HK, Maby P, Angelova M, et al. (2016) Integrative Analyses of Colorectal Cancer Show Immunoscore Is a Stronger Predictor of Patient Survival Than Microsatellite Instability, Immunity 44(3): 698-711.

33. Mlecnik B, Van den Eynde M, Bindea G, Church SE, Vasaturo A, et al. (2018) Comprehensive Intrametastatic Immune Quantification and Major Impact of Immunoscore on Survival. J Natl Cancer Inst. 110(1).

34. Turcotte S, Rosenberg SA (2011) Immunotherapy for metastatic solid cancers. Adv Surg 45: 341-360.

35. Neelapu SS, Locke FL, Bartlett NL, Lekakis LJ, Miklos DB, et al. (2017) Axicabtagene Ciloleucel CAR T-Cell Therapy in Refractory Large B-Cell Lymphoma. N Engl J Med 377(26): 2531-2544.

36. Maude SL, Laetsch TW, Buechner J, Rives S, Boyer M, et al. (2018) Tisagenlecleucel in Children and Young Adults with B-Cell Lymphoblastic Leukemia. N Engl J Med 378(5): 439-448.

37. Sackstein R, Schatton T, Barthel SR (2017) T-lymphocyte homing: an underappreciated yet critical hurdle for successful cancer immunotherapy, Lab Invest 97(6): 669-697.

38. Durgeau A, Virk Y, Corgnac S, Mami-Chouaib F (2018) Recent Advances in Targeting CD8 T-Cell Immunity for More Effective Cancer Immunotherapy, Front Immunol 9: 14.

39. Budhu S, Loike JD, Pandolfi A, Han S, Catalano G, et al. (2010) CD8+ T cell concentration determines their efficiency in killing cognate antigenexpressing syngeneic mammalian cells in vitro and in mouse tissues. J ExpMed 207(1): 223-235

40. Maude SL, Laetsch TW, Buechner J, Rives S, Boyer M, et al. (2018) Tisagenlecleucel in Children and Young Adults with B-Cell Lymphoblastic Leukemia. N Engl J Med 378(5): 439-448. 
41. Ramsay AG, Clear AJ, Fatah R, Gribben JG (2012) Multiple inhibitory ligands induce impaired T-cell immunologic synapse function in chronic lymphocytic leukemia that can be blocked with lenalidomide: establishing a reversible immune evasion mechanism in human cancer. Blood 120(7): 1412-1421.

42. Camisaschi C, Vallacchi V, Vergani E, Tazzari M, Ferro S, et al. (2016) Targeting Immune Regulatory Networks to Counteract Immune Suppression in Cancer, Vaccines 4(4).

43. Karagiannis GS, Poutahidis T, Erdman SE, Kirsch R, Riddell RH, et al (2012) Cancer-associated fibroblasts drive the progression of metastasis through both paracrine and mechanical pressure on cancer tissue. Mol Cancer Res MCR 10(11): 1403-1418.

44. Harper J, Sainson RC (2014) Regulation of the anti-tumour immune response by cancer-associated fibroblasts, Semin Cancer Biol 25: 69-77.

45. Karagiannis GS, Poutahidis T, Erdman SE, Kirsch R, Riddell RH, et al (2012) Cancer-associated fibroblasts drive the progression of metastasis through both paracrine and mechanical pressure on cancer tissue. Mol Cancer Res MCR 10(11): 1403-1418.

46. Herrera M, Herrera A, Domínguez G, Silva J, García V, et al. (2013) Cancerassociated fibroblast and M2 macrophage markers together predict outcome in colorectal cancer patients. Cancer Sci 104(4): 437-444.

47. Huber V, Camisaschi C, Berzi A, Ferro S, Lugini L, et al. (2017) Cancer acidity: An ultimate frontier of tumor immune escape and a novel target of immunomodulation, Semin Cancer Biol 43: 74-89.

48. Stephens TC, Peacock JH (1978) Cell yield and cell survival following chemotherapy of the B16 melanoma. Br J Cancer 38(5): 591-598.

49. Tran E, Longo DL, Urba WJ (2017) A Milestone for CAR T Cells. N Engl J Med 377(26): 2593-2596.

50. Yu S, Li A, Liu Q Li T, Yuan X, et al. (2017) Chimeric antigen receptor T cells: a novel therapy for solid tumors. J Hematol Oncol 10(1): 78.

51. Neelapu SS, Locke FL, Bartlett NL, Lekakis LJ, Miklos DB, et al. (2017) Axicabtagene Ciloleucel CAR T-Cell Therapy in Refractory Large B-Cell Lymphoma. N Engl J Med 377 (26): 2531-2544.

52. Schuster SJ, Svoboda J, Chong EA, Nasta SD, Mato AR, et al. (2017) Chimeric Antigen Receptor T Cells in Refractory B-Cell Lymphomas, N Engl J Med 377(26): 2545-2554.

53. Ma JS, Kim JY, Kazane SA, Choi SH, Yun HY, et al. (2016) Versatile strategy for controlling the specificity and activity of engineered T cells, Proc Natl Acad Sci USA 113(4): E450-458.

54. Rodgers DT, Mazagova M, Hampton EN, Cao Y, Ramadoss NS, et al (2016)Switch-mediated activation and retargeting of CAR-T cells for B-cell malignancies. Proc Natl Acad Sci USA 113(4): E459-468.

55. Juillerat A, Marechal A, Filhol JM, Valton J, Duclert A, et al. (2016) Design of chimeric antigen receptors with integrated controllable transient functions. Sci Rep 6: 18950.

56. Capsomidis A, Benthall G, Van Acker HH, Fisher J, Kramer AM, et al. (2018) Chimeric Antigen Receptor-Engineered Human Gamma Delta T Cells: Enhanced Cytotoxicity with Retention of Cross Presentation. Mol Ther J Am Soc Gene Ther 26(2): 354-365.

57. Mirzaei HR, Mirzaei H, Lee SY, Hadjati J, Till BG (2016) Prospects for chimeric antigen receptor (CAR) $\gamma \delta$ T cells: A potential game changer for adoptive T cell cancer immunotherapy. Cancer Lett 380(2): 413-423.

58. Abate-Daga D, Hanada K, Davis JL, Yang JC, Rosenberg SA, et al. (2013) Expression profiling of TCR-engineered T cells demonstrates overexpression of multiple inhibitory receptors in persisting lymphocytes. Blood 122(8): 1399-1410.

59. Ren J, Zhang X, Liu X, Fang C, Jiang S, et al. (2017) A versatile system for rapid multiplex genome-edited CAR T cell generation. Oncotarget 8(10): 17002-17011.
60. Yoon DH, Osborn MJ, Tolar J, Kim CJ(2018) Incorporation of Immune Checkpoint Blockade into Chimeric Antigen Receptor T Cells (CAR-Ts): Combination or Built-In CAR-T. Int J Mol Sci 19(2).

61. Rupp LJ, Schumann K, Roybal KT, Gate RE, Ye CJ, et al. (2017) CRISPR/ Cas9-mediated PD-1 disruption enhances anti-tumor efficacy of human chimeric antigen receptor T cells. Sci Rep 7(1): 737.

62. Eyquem J, Mansilla-Soto J, Giavridis T, van der Stegen SJ, Hamieh M, et al. (2017) Targeting a CAR to the TRAC locus with CRISPR/Cas9 enhances tumour rejection. Nature 43(7643): 113-117.

63. Lonez C, Verma B, Hendlisz A, Aftimos P, Awada A, et al. (2017) Study protocol for THINK: a multinational open-label phase I study to assess the safety and clinical activity of multiple administrations of NKR-2 in patients with different metastatic tumour types. BMJ Open 7(11): e017075.

64. Rosenberg SA, Restifo NP, Yang JC, Morgan RA, Dudley ME (2008) Adoptive cell transfer: a clinical path to effective cancer immunotherapy. Nat Rev Cancer 8(4): 299-308.

65. Tran E, Robbins PF, Lu YC, Prickett TD, Gartner JJ, et al. (2016) T-Cell Transfer Therapy Targeting Mutant KRAS in Cancer. N Engl J Med 375(23): 2255-2262.

66. Rosenberg SA, Yang JC, Sherry RM, Kammula US, Hughes MS, et al. (2011) Durable complete responses in heavily pretreated patients with metastatic melanoma using T-cell transfer immunotherapy. Clin Cancer Res 17(13): 4550-4557.

67. Cole DJ, Taubenberger JK, Pockaj BA, Yannelli JR, Carter C, et al. (1994) Histopathological analysis of metastatic melanoma deposits in patients receiving adoptive immunotherapy with tumor-infiltrating lymphocytes, Cancer Immunol Immunother 38(5): 299-303.

68. Dudley ME, Gross CA, Somerville RP, Hong Y, Schaub NP, et al. (2013) Randomized selection design trial evaluating CD8+-enriched versus unselected tumor-infiltrating lymphocytes for adoptive cell therapy for patients with melanoma. J Clin Oncol 31(17): 2152-2159.

69. Hinrichs CS, Rosenberg SA (2014) Exploiting the curative potential of adoptive T-cell therapy for cancer. Immunol Rev 257(1): 56-71.

70. Koller KM, Wang W, Schell TD, Cozza EM, Kokolus KM, et al. (2016) Malignant melanoma-The cradle of anti-neoplastic immunotherapy. Crit Rev Oncol Hematol 106: 25-54.

71. Aebersold P, Hyatt C, Johnson S, Hines K, Korcak L, et al. (1991) Lysis of autologous melanoma cells by tumor-infiltrating lymphocytes: association with clinical response. J Natl Cancer Inst 83(13): 932-937.

72. Schwartzentruber DJ, Hom SS, Dadmarz R, White DE, Yannelli JR, et al. (1994) In vitro predictors of therapeutic response in melanoma patients receiving tumor-infiltrating lymphocytes and interleukin-2, J Clin Oncol 12(7): 1475-1483.

73. Verdegaal EM, de Miranda NF, Visser M, Harryvan T, van Buuren MM, et al. (2016) Neoantigen landscape dynamics during human melanoma-T cell interactions. Nature vol 536(7614): 91-95.

74. Goff SL, Dudley ME, Citrin DE, Somerville RP, Wunderlich JR, et al. (2016) Randomized, Prospective Evaluation Comparing Intensity of Lymphodepletion Before Adoptive Transfer of Tumor-Infiltrating Lymphocytes for Patients With Metastatic Melanoma J Clin Oncol vol 34(20): 2389-2397.

75. Jiang SS, Tang Y, Zhang YJ, Weng DS, Zhou ZG, et al. (2015) A phase clinical trial utilizing autologous tumor-infiltrating lymphocytes in patients with primary hepatocellular carcinoma. Oncotarget 6(38): 41339-41349.

76. Farkona S, Diamandis EP, Blasutig IM (2016) Cancer immunotherapy: the beginning of the end of cancer? BMC Med 14: 73.

77. Schuler G (2010) Dendritic cells in cancer immunotherapy. Eur J Immunol 40(8): 2123-2130. 
78. Timmerman JM, Levy R (2013) Dendritic cell immunotherapy. Ann NY Acad Sci 1284: 31-45.

79. Kohlhapp FJ, Kaufman HL (2016) Molecular Pathways: Mechanism of Action for Talimogene Laherparepvec, a New Oncolytic Virus Immunotherapy, Clin Cancer Res 22(5): 1048-1054.

80. Puzanov I, Milhem MM, Minor D, Hamid O, Li A, et al. (2016) Talimogene Laherparepvec in Combination With Ipilimumab in Previously Untreated, Unresectable Stage IIIB-IV Melanoma, J Clin Oncol 34(22): 2619-2626

81. Andtbacka RHI, Kaufman HL, Collichio F, Amatruda T, Senzer N, et al. (2015) Talimogene Laherparepvec Improves Durable Response Rate in Patients With Advanced Melanoma, J Clin Oncol 33(25): 2780-2788.

82. Andtbacka RH, Ross M, Puzanov I, Milhem M, Collichio F, et al. (2016) Patterns of Clinical Response with Talimogene Laherparepvec (T-VEC) in Patients with Melanoma Treated in the OPTiM Phase III Clinical Trial. Ann Surg Oncol 23(13): 4169-4177.

83. Ribas A, Dummer R, Puzanov I, VanderWalde A, Andtbacka RHI, et al. (2017) Oncolytic Virotherapy Promotes Intratumoral T Cell Infiltration and Improves Anti-PD-1 Immunotherapy. Cell 170(6): 1109-1119 e10.

84. Belani CP, Chakraborty BC, Modi RI, Khamar BM (2017) A randomized trial of TLR-2 agonist CADI-05 targeting desmocollin-3 for advanced non-small-cell lung cancer. Ann Oncol 28(2): 298-304.

85. Khamar B (2017) Metastatic Squamous Non-Small Cell Lung Cancer: Current Status and What Next? J Cancer Clin Trials 2(3): e109.

86. Tsuta K, Tanabe Y, Yoshida A, Takahashi F, Maeshima AM, et al. (2011) Utility of 10 immunohistochemical markers including novel markers (desmocollin-3, glypican 3, S100A2, S100A7, and Sox-2) for differential diagnosis of squamous cell carcinoma from adenocarcinoma of the Lung J Thorac. Oncol 6(7): 1190-1199.

87. Khamar B (2017) Desmocollin-3 and Cancer, Biomed J Sci Tech Res 1(5): $1-5$.

88. Rakshit S, Ponnusamy M, Papanna S, Saha B, Ahmed A, et al. (2012) Immunotherapeutic efficacy of Mycobacterium indicus pranii in eliciting anti-tumor T cell responses: critical roles of IFN $\gamma$, Int J Cancer 130(4): 865-875.

89. Ahmad F, Mani J, Kumar P, Haridas S, Upadhyay P, et al. (2011) Activation of anti-tumor immune response and reduction of regulatory T cells with Mycobacterium indicus pranii (MIP) therapy in tumor bearing mice. PloS One 6(9): e25424.

90. Khamar BM, O’Donnell, Belani C (2012) Intradermal toll like receptor-2 (TLR2) agonist mycobacterium $\mathrm{w}$ (Cadi-05) in the treatment of BCG refractory non muscle invasive transitional cell carcinoma of bladder. presented at the Annual meeting of Society for Immunotherapy of Cancer (SITC), North Bethesda, MD, USA

91. Mosca P, Nair S, Ayre S, Shi W, Eid S, et al. (2010) Immunologic Therapy with Cadi-05 for the Treatment of Advanced Melanoma.

92. Khamar BM (2006) Poly-TLR agonist polyantigenic vaccine cadi-05 in advanced bladder cancer. Cancer Res 66(8 Supplement): 682.

93. Xia L, Schrump DS, Gildersleeve JC (2016) Whole-Cell Cancer Vaccines Induce Large Antibody Responses to Carbohydrates and Glycoproteins. Cell Chem Biol 23(12): 1515-1525.
94. Lipson EJ, Sharfman WH, Chen S, McMiller TL, Pritchard TS, et al. (2015) Safety and immunologic correlates of Melanoma GVAX, a GM-CSF secreting allogeneic melanoma cell vaccine administered in the adjuvant setting. J Transl Med 13: 214

95. Antonarakis ES, Eisenberger MA (2013) Phase III Trials With DocetaxelBased Combinations for Metastatic Castration-Resistant Prostate Cancer: Time to Learn From Past Experiences. J Clin Oncol 31(14): 17091712

96. Copier J, Dalgleish A (2010) Whole-cell vaccines: A failure or a success waiting to happen?. Curr Opin Mol Ther 12(1): 14-20.

97. Hodi FS, O’Day SJ, McDermott DF, Weber RW, Sosman JA, et al. (2010) Improved survival with ipilimumab in patients with metastatic melanoma. N Engl J Med 363(8): 711-723.

98. Giaccone G, Bazhenova LA, Nemunaitis J, Tan M, Juhász E, et al. (2015) A phase III study of belagenpumatucel-L, an allogeneic tumour cell vaccine, as maintenance therapy for non-small cell lung cancer, Eur J Cancer 51(16): 2321-2329.

99. Rini BI, Stenzl A, Zdrojowy R, Kogan M, Shkolnik M, et al. (2016) IMA901, a multipeptide cancer vaccine, plus sunitinib versus sunitinib alone, as first-line therapy for advanced or metastatic renal cell carcinoma (IMPRINT): a multicentre, open-label, randomised, controlled, phase 3 trial. Lancet Oncol 17(11): 1599-1611.

100. Butts C, Socinski MA, Mitchell PL, Thatcher N, Havel L, et al. (2014) Tecemotide (L-BLP25) versus placebo after chemoradiotherapy for stage III non-small-cell lung cancer (START): a randomised, doubleblind, phase 3 trial. Lancet Oncol 15(1): 59-68.

101. Katakami N, Hida T, Nokihara H, Imamura F, Sakai H, et al. (2017) Phase I/II study of tecemotide as immunotherapy in Japanese patients with unresectable stage III non-small cell lung cancer, Lung Cancer Amst Neth 105: 23-30.

102. Pujol JL, Vansteenkiste JF, De Pas TM, Atanackovic D, Reck $M$, et al. (2015) Safety and Immunogenicity of MAGE-A3 Cancer Immunotherapeutic with or without Adjuvant Chemotherapy in Patients with Resected Stage IB to III MAGE-A3-Positive Non-Small-Cell Lung Cancer. J Thorac Oncol 10(10): 1458-1467.

103. Vansteenkiste JF, Cho BC, Vanakesa T, De Pas T, Zielinski M, et al. (2016) Efficacy of the MAGE-A3 cancer immunotherapeutic as adjuvant therapy in patients with resected MAGE-A3-positive non-small-cell lung cancer (MAGRIT): a randomised, double-blind, placebo-controlled, phase 3 trial. Lancet Oncol 17(6): 822-835.

104. Kantoff PW, Schuetz TJ, Blumenstein BA, Glode LM, Bilhartz DL, et al. (2010) Overall survival analysis of a phase II randomized controlled trial of a Poxviral-based PSA-targeted immunotherapy in metastatic castration-resistant prostate cancer. J Clin Oncol 28(7): 1099-1105.

105. Quoix E, Lena H, Losonczy G, Forget F, Chouaid C, et al. (2016) TG4010 immunotherapy and first-line chemotherapy for advanced non-small-cell lung cancer (TIME): results from the phase $2 \mathrm{~b}$ part of a randomised, double-blind, placebo-controlled, phase $2 \mathrm{~b} / 3$ trial. Lancet Oncol 17(2): 212-223. 
Creative Commons Attribution 4.0 International License

For possible submissions Click Here
Submit Article

\section{Novel Approaches in Cancer Study \\ Benefits of Publishing with us}

- High-level peer review and editorial services

- Freely accessible online immediately upon publication

- Authors retain the copyright to their work

- Licensing it under a Creative Commons license

- Visibility through different online platforms 